\title{
A CHARACTER-THEORY-FREE CHARACTERIZATION OF THE MATHIEU GROUP $M_{12}$
}

\author{
DIETER HELD and JÖRG HRABĚ DE ANGELIS
}

(Received 5 July 1989)

Communicated by $\mathbf{H}$. Lausch

\begin{abstract}
The known characterization of the Mathieu group $M_{12}$ by the structure of the centralizer of a 2-central involution is based on the application of the theory of exceptional characters and uses in addition a block theoretic result which asserts that a simple group of order $\left|M_{12}\right|$ is isomorphic to $M_{12}$. The details of the proof of the latter result had never been published. We show here that $M_{12}$ can be handled in a completely elementary and group theoretical way.
\end{abstract}

1980 Mathematics subject classification (Amer. Math. Soc.) (1985 Revision): 20 D 05, 20 D 08.

The object of this paper is to present a character theory free proof of the following result.

THeOREM. Let $G$ be a finite, nonabelian simple group which possesses an involution such that its centralizer in $G$ is isomorphic to the centralizer of a 2-central involution in $M_{12}$. Then $G$ is isomorphic to $M_{12}$, the Mathieu group on 12 letters.

The main point here is as in [7], that our proof will be completely free of applications of the theory of group characters. Predecessors of this theorem are $[1$, Theorem (6A)] and [12, Theorem]. The results of both papers had been easily combined in [4] to show that the above theorem holds. However, the proofs in [1] and in [12] rely upon a theorem of $R$. G. Stanton [8] which asserts that a simple group of order 95,040 or $244,823,040$ is isomorphic to either $M_{12}$ or $M_{24}$.

(C) 1990 Australian Mathematical Society $0263-6115 / 90 \$ A 2.00+0.00$ 
As a matter of fact, the Ph.D. thesis of R. G. Stanton, written under the supervision of Richard Brauer, had not been published, and there is only the short summary [8] available in which he describes his methods, which are heavily block theoretical and computational. Thus, it seems worthwhile to present an elementary and complete proof of the charcterization of $M_{12}$ by a 2-central involution. We remark that in [6] we had shown that the characterization of $M_{24}$ of [5] can be done without referring to the result of [8]; moreover, the second author has shown that for the characterizations of $M_{22}$ and $M_{23}$, originally due to Z. Janko, one does not need recourse to the theory of exceptional characters.

\section{The centralizer of a 2-central involution in $M_{12}$}

Denote by $H$ the centralizer of a 2-central involution $z_{1}$ of $M_{12}$. According to [4], $H$ can be generated by elements $z_{1}, z_{2}, z_{3}, a, b, c, d$, where all elements except $c$ are involutions and $c$ is an element of order 3. The subgroup $E$ generated by $z_{1}, z_{2}, z_{3}$ is elementary abelian of order 8 and normal in $H$, and $\langle a, b\rangle\langle c\rangle$ is isomorphic to $A_{4}$ such that $a^{c}=b, b^{c}=a b$. The involution $d$ inverts $c$. The action on $E$ for the elements $a, b, c, d$ is described by the following matrices with entries from $G F(2)$ with respect to the basis $\left\{z_{1}, z_{2}, z_{3}\right\}$ of the "vector space" $E$ over $G F(2)$ :

$$
\begin{gathered}
a \rightarrow\left(\begin{array}{lll}
1 & 0 & 0 \\
0 & 1 & 0 \\
1 & 0 & 1
\end{array}\right), \quad b \rightarrow\left(\begin{array}{lll}
1 & 0 & 0 \\
1 & 1 & 0 \\
0 & 0 & 1
\end{array}\right), \quad a b \rightarrow\left(\begin{array}{lll}
1 & 0 & 0 \\
1 & 1 & 0 \\
1 & 0 & 1
\end{array}\right), \\
d \rightarrow\left(\begin{array}{lll}
1 & 0 & 0 \\
0 & 1 & 0 \\
0 & 1 & 1
\end{array}\right), \quad c \rightarrow\left(\begin{array}{lll}
1 & 0 & 0 \\
0 & 0 & 1 \\
0 & 1 & 1
\end{array}\right) .
\end{gathered}
$$

We have $a^{d}=z_{1} z_{2} a$ and $b^{d}=z_{1}^{\beta} z_{2} z_{3} a b$, where $\beta \in\{0,1\}$. Since $d$ inverts $c$, we get from $a^{d c d c}=a$ that $\beta=1$.

It is now a routine matter to calculate the conjugacy clases of $H$. The results are listed in Table I.

\section{The fusion of involutions and the possible orders for $G$}

In what follows, $G$ denotes a finite simple group possessing an involution $z$ such that $\mathrm{C}(z)$ is isomorphic to $H$. We put $\mathrm{C}(z)=H$ and use the notation developed for $H$ so far. Thus, in particular, $z=z_{1}$, and since the center of a $S_{2}$-subgroup of $H$ is cyclic, we see that $z_{1}$ is 2-central in $G$. 
TABLE I

\begin{tabular}{ccccc}
\hline$x$ & $x^{2}$ & $o(x)$ & $\mathbf{C}_{H}(x)$ & $\left|c c l_{H}(x)\right|$ \\
\hline 1 & & 1 & $H$ & 1 \\
$z_{1}$ & & 2 & $H$ & 1 \\
$z_{2}$ & & 2 & $E\langle a, d\rangle$ & 6 \\
$a$ & & 2 & $\left\langle z_{1}, z_{2}, a, b\right\rangle$ & 12 \\
$z_{3} a$ & $z_{1}$ & 4 & $\left\langle z_{3} a, z_{2}, b, z_{3} d\right\rangle$ & 6 \\
$z_{2} z_{3} a$ & $z_{1}$ & 4 & $\left\langle z_{2} z_{3} a, z_{2}, z_{3} b, z_{3} d\right\rangle$ & 6 \\
$c$ & & 3 & $\left\langle z_{1}, c\right\rangle$ & 32 \\
$d$ & & 2 & $\left\langle z_{1}, z_{2}, d\right\rangle$ & 24 \\
$z_{3} d$ & $z_{2}$ & 4 & $\left\langle z_{1}, z_{3} a, z_{3} d\right\rangle$ & 12 \\
$z_{1} z_{3} d$ & $z_{2}$ & 4 & $\| l$ & 12 \\
$b d$ & $z_{2} z_{3} a$ & 8 & $\langle b d\rangle$ & 24 \\
$z_{3} b d$ & $z_{1} z_{3} a$ & 8 & $\left\langle z_{3} b d\right\rangle$ & 24 \\
$z_{1} c$ & & 6 & $\left\langle z_{1}, c\right\rangle$ & 32 \\
& & & & 192 \\
\hline
\end{tabular}

(2.1) LemMA. $A S_{2}$-subgroup of $\mathrm{C}(a)$ has order $2^{4}$.

Proof. We have $\mathbf{C}_{H}(a)=\langle a\rangle \times\left\langle z_{1}, z_{2}, b\right\rangle$. Thus $\mathbf{C}_{H}(a)^{\prime}=\left\langle z_{1}\right\rangle$. The assertion follows.

(2.2) Lemma. If $z_{1} \sim z_{2}$ in $G$, then $O(\mathbf{C}(a))=\langle 1\rangle$.

Proof. Put $K=O(\mathbf{C}(a))$ and act with the four-group $\left\langle z_{1}, z_{2}\right\rangle$ on $K$. If $x \in\left\langle z_{1}, z_{2}\right)^{\#}$, then $\mathbf{C}(a) \cap \mathbf{C}(x)$ is a 2-group by the structure of $H$, and so, $x$ operates fixed-point-freely on $K$. Application of $[9,5.1 .9]$ yields that $\left[K, z_{1} z_{2}\right]=\langle 1\rangle$. We conclude that $K=\langle 1\rangle$.

(2.3) LemmA. If $z_{1} \sim z_{2}$ in $G$, then $\mathbf{C}(a)=\langle a\rangle \times U$, where $U$ possesses a subgroup of index 2.

Proof. A result of W. Gaschütz yields the existence of a subgroup $U$ such that $\mathbf{C}(a)=\langle a\rangle \times U$. Clearly, $P=\left\langle a, z_{1}, z_{2}, b\right\rangle \cap U$ is dihedral of order 8 with center equal to $z_{1}$ as $\mathbf{C}_{H}(a)^{\prime}=\left\langle z_{1}\right\rangle$. We have that a $S_{2}$-subgroup of $U$ is selfnormalizing in $U$. Application of a result of $O$. Grün [3, 7.4.2] yields that $P \cap U^{\prime}$ is contained in $\left\langle z_{1}, z_{2}\right\rangle$; note that the elements of $\left\langle z_{1}, z_{2}\right\rangle^{\#}$ are the only $G$-conjugates of $z_{1}$ in $\left\langle a, z_{1}, z_{2}, b\right\rangle$. Now, from $[3,7.3 .1]$ it follows that $U$ has a subgroup of index 2; use the fact that $P / P \cap U^{\prime} \cong U / U^{*}$ for some normal subgroup $U^{*}$ of $U$. The lemma is proved. 
(2.4) Lemma. The normalizer of $E$ in $G$ is $H$.

Proof. By way of contradiction assume that $\mathbf{N}(E) \supset H$. Then $\mathbf{N}(E) / E \cong$ $G L(3,2)$; note that $E$ is selfcentralizing. Thus, $z_{1} \sim z_{2}$ and $a \sim d$ hold in $\mathbf{N}(E)$. It follows that $G$ possesses precisely two classes of involutions. The representatives of these classes are $z_{1}$ and $a$.

From (2.3) we know that $\mathbf{C}(a)=\langle a\rangle \times U$, where $U$ possesses a subgroup $U_{1}$ of index 2, and from (2.2) we get that $O(\mathbf{C}(a))=\langle 1\rangle$. If a $S_{2}$-subgroup of $U_{1}$ is cyclic then $U$ is a 2-group by transfer results, and in this case $\mathbf{C}(a)=$ $\mathbf{C}_{H}(a)$. Assume that a $S_{2}$-subgroup $X$ of $U_{1}$ is a four-group. If $\mathbf{C}_{H}(a) \subset$ $\mathbf{C}(a)$, then all involutions of $U_{1}$ are conjugate in $U_{1}$ as otherwise $U_{1}$ would possess a normal 2-complement which is $\langle 1\rangle$ by (2.2). It follows that $\left(\mathbf{N}(X) \cap U_{1}\right) /\left(\mathbf{C}(X) \cap U_{1}\right)$ has order 3. Clearly, the commutator subgroup of $\mathbf{C}(a)$ lies in $U_{1}$. Since $\mathbf{C}_{H}(a)^{\prime}=\left\langle z_{1}\right\rangle$, we get $z_{1} \in U_{1}$ and this implies that $\left\langle z_{1}, z_{2}\right\rangle$ is a $S_{2}$-subgroup of $U_{1}$. Thus, $\mathbf{C}(X) \cap U_{1}=X$ and $\left|\mathbf{N}(X) \cap U_{1}\right|=2^{2} \cdot 3$. Note that $\left\langle z_{1}, z_{2}\right\rangle=\mathbf{Z}(E\langle a, d\rangle)$. Since $\mathbf{N}(E) / E \cong$ $G L(3,2)$, we see that $\mathbf{N}\left(\left\langle z_{1}, z_{2}\right\rangle\right)$ lies in $\mathbf{N}(E)$ and has order $2^{6} \cdot 3$. Thus $\mathbf{N}\left(\left\langle z_{1}, z_{2}\right\rangle\right) \cap U_{1} \subseteq \mathbf{N}(E)$. But then $a$ would centralize an element of order 3 in $\mathbf{N}(E)$ which contradicts the structure of $G L(3,2)$. We have shown that $\mathbf{C}(a)=\mathbf{C}_{H}(a)$.

We know that $G$ has precisely two classes of involutions and that if $x$ is an involution of $\mathbf{N}(E)$ then $\mathbf{C}(x) \subseteq \mathbf{N}(E)$. Application of [10, Lemma 5.35] yields $\mathrm{N}(E)=G$ which contradicts the simplicity of $G$. The assertion is proved.

(2.5) LemMa. The involution $z_{1}$ is conjugate in $G$ to an element of $H \backslash\left\langle z_{1}\right\rangle$.

Proof. Assume by way of contradiction that $G$ is 2-normal. Put $T=$ $E\langle a, b, d\rangle$. Since $\mathbf{Z}(T)=\left\langle z_{1}\right\rangle$ and $T \in \operatorname{Syl}_{2}(G)$, we get from $O$. Grün's theorem [3, 7.5.2] that $T \cap G^{\prime}=T \cap H^{\prime}$. It would follow that $G$ had a normal subgroup of index 2 . Therefore $G$ is not 2-normal. This implies the existence of an element $g$ in $G$ such that $z_{1} \in T \cap T^{g}$, but $\left\langle z_{1}\right\rangle \neq \mathbf{Z}\left(T^{g}\right)$. The center of $T^{g}$ is $\left\langle z_{1}^{g}\right\rangle$, and so, $z_{1} \neq z_{1}^{g}$. Since $z_{1} \in T^{g}$, we have $\left[z_{1}, z_{1}^{g}\right]=1$. It follows $z_{1}^{g} \in H \backslash\left\langle z_{1}\right\rangle$.

(2.6) Lemma. In $G$ we have $z_{1} \sim z_{2} \sim d$ and a is not conjugate to $z_{1}$.

Proof. We know that $\mathrm{N}(E)=H$ and that $z_{1}$ is not conjugate to $a$ in $G$; remember that a $S_{2}$-subgroup of $\mathrm{C}(a)$ has order $2^{4}$. 
By way of contradiction we suppose that $d \sim a$ holds in $G$. From (2.5) we get $z_{1} \sim z_{2}$. In $\langle a\rangle \times\left\langle z_{1}, z_{2}, b\right\rangle$ there are precisely two elementary abelian subgroups of order 8 . Thus, as $d \sim a$, there are the following two possibilities:

$$
\begin{gathered}
\left\langle a, z_{1}, z_{2}\right\rangle \sim\left\langle d, z_{1}, z_{2}\right\rangle ; \\
\left\langle a, z_{1}, b\right\rangle \sim\left\langle d, z_{1}, z_{2}\right\rangle .
\end{gathered}
$$

The possibility (ii) does not occur as in the group on the left there is only one $G$-conjugate of $z_{1}$ whereas in the group on the right there are three $G$-conjugates of $z_{1}$. Thus, we are in case (i). We get that the conjugation (i) is performed by an element of $\mathbf{N}\left(\left\langle z_{1}, z_{2}\right\rangle\right)$. Denote the latter group by $N$. Our assumptions imply that $|N|=2^{6} \cdot 3$, since $\left\langle z_{1}, z_{2}\right\rangle=\mathbf{Z}(E\langle a, d\rangle)$. Clearly, $O_{2}(N)=E\langle a, d\rangle=\mathbf{C}\left(\left\langle z_{1}, z_{2}\right\rangle\right)$. By a result of Baer-Suzuki [3, 3.8.2] there is an element $k$ of order 3 in $N$ which is inverted by $b$. We may assume that $k: z_{1} \rightarrow z_{2} \rightarrow z_{1} z_{2}$. The group $\langle k, b\rangle \cong \Sigma_{3}$ acts on $E\langle a, d\rangle /\left\langle z_{1}, z_{2}\right\rangle$. Clearly, $k \notin \mathbf{N}(E)$ as $\mathbf{N}(E)=H$. We have $o\left(z_{3}\right)=$ $o(a)=o(d)=o\left(z_{3} a d\right)=2$ and $o\left(z_{3} a\right)=o\left(z_{3} d\right)=o(a d)=4$. Since $k$ acts fixed-point-freely on $\left\langle z_{1}, z_{2}\right\rangle$, we see that $k$ does not leave invariant any of the following cosets: $\left\langle z_{1}, z_{2}\right\rangle z_{3} a,\left\langle z_{1}, z_{2}\right\rangle z_{3} d,\left\langle z_{1}, z_{2}\right\rangle a d$. It follows that

$$
k:\left\langle z_{1}, z_{2}\right\rangle z_{3} a \rightarrow\left\langle z_{1}, z_{2}\right\rangle z_{3} d \rightarrow\left\langle z_{1}, z_{2}\right\rangle a d .
$$

Furthermore, $k:\left\langle z_{1}, z_{2}\right\rangle z_{3} \rightarrow\left\langle z_{1}, z_{2}\right\rangle a$ and $k:\left\langle z_{1}, z_{2}\right\rangle z_{3} \rightarrow\left\langle z_{1}, z_{2}\right\rangle d$ are both impossible as $z_{1} \sim z_{2} \sim z_{3}, a \sim d$, and $a$ is not conjugate to $z_{1}$. But also $k:\left\langle z_{1}, z_{2}\right\rangle z_{3} \rightarrow\left\langle z_{1}, z_{2}\right\rangle z_{3}$ ad cannot hold as $E a d \sim E d$ in $H$ and all involutions of $E d$ are conjugate. We have obtained a contradiction which shows that $d$ cannot be conjugate to $a$ in $G$.

Thompson's transfer theorem [10,5.38] gives $d \sim z_{2}$ or $d \sim z_{1}$. Suppose that $z_{1}$ is not conjugate to $z_{2}$ in $G$. Application of (2.5) yields that $z_{1} \sim d$ in $G$. Clearly, $z_{2}$ is not conjugate to $a$ in $G$ by (2.1). Thus, in $G$ there are precisely three classes of involutions; representatives of these classes are $z_{1}, z_{2}, a$. There is an element $x$ in $\mathbf{C}(d)$ normalizing $\left\langle z_{1}, z_{2}, d\right\rangle$ such that $x^{2} \in\left\langle z_{1}, z_{2}, d\right\rangle$ and $x \notin\left\langle z_{1}, z_{2}, d\right\rangle$. Note that all elements of $\left\langle z_{1}, z_{2}\right\rangle d$ are conjugate, that $z_{1} \sim d$, and that $z_{2} \sim z_{1} z_{2} \nsim z_{1}$. It follows that $x$ centralizes $z_{1}$; this, however, is not possible. The contradiction shows that $z_{1} \sim z_{2} \sim d \nsim a$. The lemma is proved.

(2.7) Lemma. $\mathrm{C}(a)=\langle a\rangle \times L$, where $L \cong \Sigma_{4}$ or $L \cong \Sigma_{5}$. The group $\left\langle z_{1}, z_{2}\right\rangle\langle\gamma\rangle$ is isomorphic to $A_{4}$ and lies in $L^{\prime} ;$ here, $\gamma$ is an element of order 3 of $N=\mathbf{N}\left(\left\langle z_{1}, z_{2}\right\rangle\right)$. 
Proof. We have $\mathbf{C}_{H}\left(z_{2}\right)=E\langle a, d\rangle$ and $\mathbf{Z}\left(E(\langle a, d\rangle)=\left\langle z_{1}, z_{2}\right\rangle\right.$. As $z_{1} \sim z_{2} \sim z_{1} z_{2}$, we get that $N=\mathbf{N}\left(\left\langle z_{1}, z_{2}^{\prime}\right\rangle\right)$ has order $2^{6} \cdot 3$. Since $b \notin O_{2}(N)$, we get from the result of Baer-Suzuki that there is an element $k$ of order 3 in $N$ which is inverted by $b$. We know that $\mathrm{N}(E)=H$, and this implies that $E$ is not normal in $N$. Hence, $\left[k, z_{3}\right] \notin\left\langle z_{1}, z_{2}\right\rangle$. Obviously, $\left\langle z_{1}, z_{2}\right\rangle z_{3}$ cannot be mapped onto $\left\langle z_{1}, z_{2}\right\rangle a$ as all involutions of $E$ are conjugate and $a$ is not conjugate to $z_{1}$. Via $b$, the cosets $E d$ and $E z_{3} a d$ are conjugate; so, all involutions of $E d$ and $E a d$ are conjugate to $z_{1}$ in $G$. It follows that $\left\langle z_{1}, z_{2}\right\rangle a$ is kept fixed under the action of $k$ in $N$. Therefore, $k$ centralizes a conjugate of $a$ in $\left\langle z_{1}, z_{2}\right\rangle a$. Clearly, $\left\langle z_{1}\right\rangle$ is a $S_{2}$-subgroup of $\mathbf{C}(c)$. If $\langle k\rangle$ was conjugate to $\langle c\rangle$ in $G$, then we would get $a \sim z_{1}$, which is impossible. We have shown that in $G$ there are at least two classes of elements of order 3 and that 3 divides $|\mathbf{C}(a)|$; note that $c \sim c^{-1}$ in $H$.

Denote by $\gamma$ an element of order 3 of $N$ which centralizes $a$; there is such an element as all elements of $\left\langle z_{1}, z_{2}\right\rangle a$ are conjugate by the action of $\left\langle z_{3}, d\right\rangle$. Let $H_{2}=\mathrm{C}(a)$. We have that $H_{2} \supseteq\left\langle a, z_{1}, z_{2}, \gamma, b\right\rangle$ and that a $S_{2}$-subgroup of $H_{2}$ is of type $Z_{2} \times D_{8}$. Since $\gamma \in N$, we get $\left\langle z_{1}, z_{2}\right\rangle\langle\gamma\rangle \cong A_{4}$. We know from (2.1) and (2.3) that $H_{2}=\langle a\rangle \times L$ and that a $S_{2}$-subgroup of $L$ is dihedral of order 8. Moreover, $L$ possesses a subgroup $L_{1}$ of index 2. Obviously, $\left\langle z_{1}, z_{2}\right\rangle\langle\gamma\rangle \subseteq L_{1}$. Note that if $x \in\left\langle z_{1}, z_{2}\right\rangle^{\#}$ then $\mathrm{C}(x) \cap L_{1}=\left\langle z_{1}, z_{2}\right\rangle$.

Let us assume that $\left\langle z_{1}, z_{2}\right\rangle\langle\gamma\rangle \subset L_{1}$. As $O\left(L_{1}\right)$ is characteristic in $L_{1}$ and hence normal in $H_{2}$, we get $O\left(L_{1}\right)=\langle 1\rangle$. Since $\mathbf{C}\left(z_{1}\right) \cap L_{1}=\left\langle z_{1}, z_{2}\right\rangle$, there is no normal 2-subgroup of $L_{1}$ different from $\langle 1\rangle$. Denote by $K$ a minimal normal subgroup of $L_{1}$. Then $K$ has even order and is a simple group. It follows from [9, p. 129] that $K \cong A_{5}$. Thus, as $K$ is normal in $L_{1}$, we get $L_{1}=K$. It follows $L \cong \Sigma_{5}$. Clearly, if $L_{1} \cong A_{4}$ then $L \cong \Sigma_{4}$. The lemma is proved.

In what follows we shall make use of J. G. Thompson's order formula [9, 5.1.7]. Thus, if $x$ is an involution of $G$, we denote by $a(x)$ the number of pairs $(u, v)$ such that $u \sim z_{1}, v \sim a$, and $x \in\langle u v\rangle$.

(2.8) LemmA. The integer $a\left(z_{1}\right)$ is equal to 240.

Proof. The roots of $z_{1}$ lie in the $H$-classes with the representatives $z_{1}, z_{3} a$, $z_{2} z_{3} a, b d, z_{3} b d$, and $z_{1} c$.

Assume that $u v=z_{1}$. Then $u=z_{1} v$ and $v$ is conjugate to $a$ in $H$. But $z_{1} a \sim a$ in $H$ and this shows that $u v=z_{1}$ is not possible. 
Assume that $o(u v)=4$. Then $u v u v=z_{1}$, and so $u^{v}=z_{1} u$ which implies that $v \in \mathbf{N}_{H}\left(\left\langle z_{1}, u\right\rangle\right) \backslash \mathbf{C}\left(\left\langle z_{1}, u\right\rangle\right)$. First, we handle the case $u=$ $z_{2}$. We have $\mathbf{C}\left(\left\langle z_{1}, z_{2}\right\rangle\right)=E\langle a, d\rangle$, and therefore, $v$ is an involution of the coset $E\langle a, d\rangle b$. The relevant cosets with respect to $E$ containing $v$ are $E b, E a b$. In $E a \cup E a b$ there are 8 involutions conjugate to $a$. Thus, if $u=z_{2}$, there are 8 possibilities for $v$. But $z_{2}$ has precisely 6 conjugates in $H$, and therefore we get that there are $8 \cdot 6=48$ pairs $(u, v)$ such that $o(u v)=4$ and $u \sim z_{2}$ in $H$. Now assume that $u=d$. Thus $v \in\left\langle z_{1}, z_{2}, d\right\rangle z_{3} a$. This coset contains precisely four involutions; these involutions form $\left\langle z_{1}, z_{2}\right\rangle d z_{3} a$; but all these involutions are conjugate to $z_{1}$ in $G$ and we cannot find such a $v$. Thus, if $o(u v)=4$, the number of pairs $(u, v)$ such that $z_{1} \in\langle u, v\rangle$ is equal to 48 .

Assume that $o(u v)=6$. There is only one class of elements of order 6 in $H$; a representative for the class is $z_{1} c$. We have $\mathbf{C}_{H}\left(z_{1} c\right)=\left\langle z_{1}\right\rangle \times\langle c\rangle$. Thus $\mathbf{N}_{H}\left(\left\langle z_{1} c\right\rangle\right)=\left\langle z_{1}\right\rangle \times\langle c, d\rangle$. Let $u v=z_{1} c$. Then $v \sim a$ in $H$ and $v$ inverts $c$. This is not possible as all involutions of $\left\langle z_{1}, d\right\rangle$ are conjugate to $z_{1}$ in $G$. We have shown that $o(u v)=6$ is not possible.

Finally, we handle the case $o(u v)=8$. In $H$ there are exactly two classes of elements of order 8; they are represented by $b d$ and $z_{3} b d$. The elements of $\langle b d\rangle$ are $1, b d, z_{2} z_{3} a, z_{2} z_{3} a b d, z_{1}, z_{1} b d, z_{1} z_{2} z_{3} a, z_{1} z_{2} z_{3} a b d$. The involutions inverting $b d$ lie all in $\langle b d\rangle d$ and are the following elements: $d, b, z_{2} z_{3} a d, z_{2} z_{3} a b, z_{1} d, z_{1} b, z_{1} z_{2} z_{3} a d, z_{1} z_{2} z_{3} a b$. Let $u v=b d$. Then $u b d=v$. We see that there are precisely four pairs $(u, v)$ such that $u v=b d$. Thus there are precisely $4 \cdot 24=96$ pairs $(u, v)$ such that $u v$ lies in $c c l_{H}(b d)$. Let $u v=z_{3} b d$. The elements of $\left\langle z_{3} b d\right\rangle$ are $1, z_{3} b d$, $z_{1} z_{3} a, a b d, z_{1}, z_{1} z_{3} b d, z_{3} a, z_{1} a b d$. The involutions inverting $z_{3} b d$ are $d, z_{3} b, z_{1} z_{3} a d, a b, z_{1} d, z_{1} z_{3} b, z_{3} a d, z_{1} a b$. We have $u z_{3} b d=v$, and it is easy to compute that there are precisely four pairs $(u, v)$ such that $u v=z_{3} b d$. Hence, there are precisely $4 \cdot 24=96$ pairs $(u, v)$ such that $u v \in c c l_{H}\left(z_{3} b d\right)$. It follows that $a\left(z_{1}\right)=48+96+96=240$.

LEMMA. If $L \cong \Sigma_{4}$ then $a(a)=3$.

Proof. From (2.7) we get $\mathbf{C}(a)=\left\langle a, z_{1}, z_{2}, \gamma, b\right\rangle$ and $\left\langle z_{1}, z_{2}\right\rangle\langle\gamma\rangle \cong$ $A_{4}$. The conjugacy classes of $\mathrm{C}(a)$ are listed in Table II.

Put $H_{2}=\mathrm{C}(a)$. Roots of the involution $a$ are in the $H_{2}$-classes with the representatives $a$ and $a \gamma$.

All involutions of $H_{2}$ conjugate to $z_{1}$ in $G$ are conjugate to $z_{1}$ and $H_{2}$. It follows that there are precisely three pairs $(u, v)$ such that $u v=a$.

Assume that $u v=a \gamma$. Clearly, $u$ and $v$ both invert $\gamma$, and $\mathbf{N}(\langle\gamma\rangle) \cap \mathrm{H}_{2}=$ $(\langle a\rangle \times\langle\gamma\rangle)\left\langle b^{\prime}\right\rangle$, where $b^{\prime}$ is an involution conjugate to $a$ and $G$ and inverting 
TABLE II

\begin{tabular}{cccc}
\hline$x$ & $o(x)$ & $\mathbf{C}_{\mathbf{C}(a)}(x)$ & $\left|c c l_{\mathbf{C}(a)}(x)\right|$ \\
\hline 1 & 1 & $\left\langle a, z_{1}, z_{2}, \gamma, b\right\rangle$ & 1 \\
$a$ & 2 & $\|$ & 1 \\
$z_{1}$ & 2 & $\left\langle a, z_{1}, z_{2}, b\right\rangle$ & 3 \\
$a z_{1}$ & 2 & $\|$ & 3 \\
$\gamma$ & 3 & $\langle a\rangle \times\langle\gamma\rangle$ & 8 \\
$a \gamma$ & 6 & $\|$ & 8 \\
$b$ & 2 & $\left\langle a, z_{1}, b\right\rangle$ & 6 \\
$a b$ & 2 & $\|$ & 6 \\
$b z_{2}$ & 4 & $\left\langle a, b z_{2}\right\rangle$ & 6 \\
$a b z_{2}$ & 4 & $\|$ & 6 \\
& & & $\frac{48}{48}$ \\
\hline
\end{tabular}

$\gamma$. The involutions in $\left\langle z_{1}, z_{2}\right\rangle$ cannot invert $\gamma$. Thus, there is no pair $(u, v)$ such that $u v=a \gamma$. we have proved that $a(a)=3$.

(2.10) LEMMA. If $L \cong \Sigma_{4}$, then $|G|=2^{6} \cdot 3^{3} \cdot 7$.

Proof. We apply Thompson's order-formula and compute

$$
\begin{aligned}
|G| & =\left|\mathbf{C}\left(z_{1}\right)\right| \cdot a(a)+|\mathbf{C}(a)| \cdot a\left(z_{1}\right) \\
& =192 \cdot 3+48 \cdot 240=12,096 .
\end{aligned}
$$

(2.11) Lemma. If $L \cong \Sigma_{5}$ then $a(a)=195$.

Proof. As we have remarked in (2.7), the group $\left\langle z_{1}, z_{2}\right\rangle\langle\gamma\rangle$ is isomorphic to $A_{4}$ and lies in $L^{\prime}$ which is isomorphic to $A_{5}$. We know that $\left\langle a, z_{1}, z_{2}, b\right\rangle \in \operatorname{Syl}_{2}(\mathbf{C}(a))$. The element $\gamma$ of order 3 is centralized by an involution $b^{\prime}$ of $L \backslash L^{\prime}$, and it is clear that $b^{\prime} \sim a$ in $G$. Denote by $w$ an element of order 5 of $L$. The conjugacy classes of $C(a)$ are listed in Table III.

Put $\mathrm{H}_{2}=\mathrm{C}(a)$. The roots of $a$ lie in the $\mathrm{H}_{2}$-classes with the representatives $a, a \gamma, a w$.

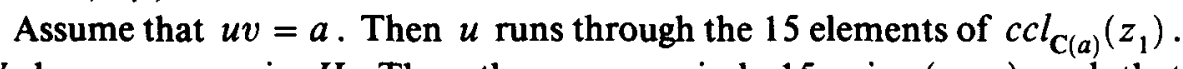
We have $z_{1} a \sim a$ in $H$. Thus, there are precisely 15 pairs $(u, v)$ such that $o(u v)=2$ and $a \in\langle u v\rangle$.

Assume that $u v=a \gamma$. Clearly, $u$ and $v$ both invert $\gamma$. We have $\mathbf{C}(\gamma) \cap$ $H_{2}=\left\langle a, b^{\prime}, \gamma\right\rangle$, and there is an involution $z$ conjugate to $z_{1}$ in $L^{\prime}$ which inverts $\gamma$ and centralizes $\left\langle a, b^{\prime}\right\rangle$. Thus, $\mathbf{N}(\langle\gamma\rangle) \cap H_{2}=\left(\left\langle a, b^{\prime}\right\rangle \times\langle\gamma\rangle\right)\langle z\rangle$. 
TABLE III

\begin{tabular}{cccc}
\hline$x$ & $o(x)$ & $\mathbf{C}_{\mathbf{C}(a)}(x)$ & $\left|c c l_{\mathbf{C}(a)}(x)\right|$ \\
\hline 1 & 1 & $\langle a\rangle \times L$ & 1 \\
$a$ & 2 & $\|$ & 1 \\
$z_{1}$ & 2 & $\left\langle a, z_{1}, z_{2}, b\right\rangle$ & 15 \\
$\gamma$ & 3 & $\left\langle a, b^{\prime}, \gamma\right\rangle$ & 20 \\
$w$ & 5 & $\langle a, w\rangle$ & 24 \\
$b^{\prime} \gamma$ & 6 & $\left\langle a, b^{\prime}, \gamma\right\rangle$ & 20 \\
$b^{\prime} \sim a$ & 2 & $\left\langle a, z_{1}^{\prime}, b^{\prime}, \gamma\right\rangle, z_{1}^{\prime} \sim z_{1}$ & 10 \\
$z_{2} b$ & 4 & $\left\langle a, z_{2} b\right\rangle$ & 30 \\
$a z_{1}$ & 2 & $\left\langle a, z_{1}, z_{2}, b\right\rangle$ & 15 \\
$a \gamma$ & 6 & $\left\langle a, b^{\prime}, \gamma\right\rangle$ & 20 \\
$a w$ & 10 & $\langle a, w\rangle$ & 24 \\
$a b^{\prime} \gamma$ & 6 & $\left\langle a, b^{\prime}, \gamma\right\rangle$ & 20 \\
$a b$ & 2 & $\left\langle a, z_{1}, b, \gamma^{\prime}\right\rangle, \gamma^{\prime} \sim \gamma$ & 10 \\
$a z_{2} b$ & 4 & $\left\langle a, z_{2} b\right\rangle$ & 30 \\
& & & 240 \\
\hline
\end{tabular}

There are precisely 12 involutions in $\mathrm{N}(\langle\gamma\rangle) \cap H_{2}$ which invert $\gamma$ but only three of which are conjugate to $z_{1}$ in $G$. These are $z, \gamma z, \gamma^{-1} z$. We have $z \cdot a \gamma^{-1} z=a \gamma, \gamma z \cdot a z=a \gamma, \gamma^{-1} z \cdot a \gamma z=\gamma^{-1} a \gamma^{-1}=a \gamma$. It follows that there are precisely $3 \cdot 20=60$ pairs $(u, v)$ such that $o(u v)=6$ and $a \in\langle u v\rangle$.

Assume finally that $u v=a w$; thus, $o(u v)=10$. Clearly, $u$ and $v$ both invert $w$. We have $\mathbf{C}(w) \cap H_{2}=\langle a, w\rangle$. Thus, $u, v \in \mathbf{C}^{*}(w) \cap H_{2}=$ $(\langle a\rangle \times\langle w\rangle)\langle z\rangle$, where $z \in L^{\prime}$ and $z w z=w^{-1}$. As $z \in L^{\prime}$, we have $z \sim z_{1}$ in $L^{\prime}$. The involutions of $C^{*}(w) \cap H_{2}$ conjugate to $z_{1}$ in $G$ are precisely the five elements in $\langle w\rangle z$. Clearly, $w^{i} z \cdot x=a w$ has the solution $x=$ $z^{-1} w^{-i} a w$ and $x \sim a$ in $G$. It follows that there are precisely $5 \cdot 24=120$ pairs $(u, v)$ such that $o(u v)=10$. We conclude $a(a)=15+60+120=195$. The lemma is proved.

(2.12) LemMA. If $L \cong \Sigma_{5}$ then $|G|=\left|M_{12}\right|$.

Proof. Compute

$$
\begin{aligned}
\left|C\left(z_{1}\right)\right| \cdot a(a)+|\mathbf{C}(a)| \cdot a\left(z_{1}\right) & =192 \cdot 195+240 \cdot 240 \\
& =95,040=2^{6} \cdot 3^{3} \cdot 5 \cdot 11 .
\end{aligned}
$$




\section{The case $L \cong \Sigma_{4}$}

We shall show that the group $G$ with the title property does not exist.

Remember that $G$ has at least two classes of elements of order 3, namely those represented by $c$ and $\gamma$. Since $\left\langle z_{1}\right\rangle$ is a $S_{2}$-subgroup of $\mathbf{C}(c)$, and $\langle a\rangle$ is one of $\mathbf{C}(\gamma)$, we see that both $\mathbf{C}(c)$ and $\mathbf{C}(\gamma)$ have normal 2-complements. Note that $\left\langle z_{1}, d\right\rangle$ is a $S_{2}$-subgroup of $\mathbf{N}(\langle c\rangle)$ and $\left\langle a, b^{\prime}\right\rangle$ is a $S_{2}$-subgroup of $\mathbf{N}(\langle\gamma\rangle)$.

(3.1) Lemma. We have $\mathrm{C}(c)=K_{c}\left\langle z_{1}\right\rangle$, where $K_{c}$ is a normal subgroup of $\mathbf{C}(c)$ of order $3^{2}$ or $3^{3}$, and $\mathbf{N}(\langle c\rangle)=K_{c}\left\langle z_{1}, d\right\rangle$. Also, $\mathbf{C}(\gamma)=K_{\gamma}\langle a\rangle$, where $K_{\gamma}$ is a normal subgroup of $\mathbf{C}(\gamma)$ of order $3^{2}$ or $3^{3}$, and $\mathbf{N}(\langle\gamma\rangle)=K_{\gamma}\left(a, b^{\prime}\right\rangle$.

Proof. By a transfer result $[3,7.4 .5]$ both $\mathbf{C}(c)$ and $\mathbf{C}(\gamma)$ have normal 2complements. These normal 2-complements are normalized by four-groups. Consider for instance $\mathrm{C}(c)$. Put $K=K_{c}$. Then $K \unlhd K\left\langle z_{1}, d\right\rangle$. The Frattini argument together with $[9,5.1 .9]$ yields that $K$ is a 3-group. As $3^{3}$ divides $|G|$, we get that $|K| \in\left\{3^{2}, 3^{3}\right\}$.

(3.2) Lemma. Denote by $T$ a $S_{3}$-subgroup and by $S$ a $S_{7}$-subgroup of $G$. Then $|G: \mathbf{N}(S)| \in\{64,288\}$ and $|G: \mathbf{N}(T)| \in\{112,448\}$. If $|G: \mathbf{N}(S)|=$ 288 , then $\mathrm{C}(S)=S . A S_{3}$-subgroup of $\mathrm{G}$ is not cyclic.

Proof. From the order of $G$ we get that $G$ has no proper subgroup of index smaller than 9. Thus, from Sylow's theorem, we get $|G: \mathbf{N}(S)| \in$ $\{36,64,288\}$ and $|G: \mathbf{N}(T)| \in\{16,28,64,112,488\}$. If $|G: \mathbf{N}(S)|=$ 36 , then $|\mathbf{N}(S)|=2^{4} \cdot 3 \cdot 7$; but then an involution would centralize an element of order 7 which is not the case.

Clearly, $T$ is not cyclic, since $\langle c\rangle$ is not conjugate to $\langle\gamma\rangle$ in $G$. If we had $|G: \mathbf{N}(T)| \in\{16,64\}$, then an element of order 7 in $G$ would centralize a Sylow 3-subgroup which contradicts (3.1).

Suppose that $|G: \mathbf{N}(T)|=28$. Then $|\mathbf{N}(T)|=2^{4} \cdot 3^{3}$. Assume that $T^{\prime} \neq\langle 1\rangle$. Then $\left|T^{\prime}\right|=3$ and an element of order 3 of $G$ is centralized by a group of order 8 which is not possible. Thus $T^{\prime}=\langle 1\rangle$. If $T$ was of type $(3,9)$, then $T$ had a characteristic subgroup of order 3 , and again we get a contradiction to the structures of centralizers of involutions in $G$. It follows that $T$ is elementary abelian. From (3.1) we get $|\mathbf{C}(c)|=|\mathbf{C}(\gamma)|=2 \cdot 3^{3}$. Therefore, in $\mathbf{N}(T)$ there are 8 conjugates of $c$ and 8 conjugates of $\gamma$. By a lemma of Burnside [3,7.1.1], there is an element $x$ of order 3 in $T$ which is not conjugate to $c$ and not to $\gamma$ in $G$. Thus, $x$ is not centralized by an 
involution. It follows that $x$ has 16 conjugates in $\mathbf{N}(T)$; but $8+8+16+1>$ $|T|=27$. We have obtained a contradiction also in this case.

Assume finally that $|G: \mathbf{N}(S)|=288$. Then $|\mathbf{N}(S)|=2 \cdot 3 \cdot 7$. Let $x$ be an element of order 3 in $\mathbf{C}(S)$. Then, the order of $\mathbf{C}(x)$ is either $3^{2} .7$ or $3^{3} .7$; note, that by (3.1), the element $x$ cannot be centralized by an involution. This contradicts the order of $\mathbf{N}(S)$. The lemma is proved.

We shall now rule out all four cases of Lemma (3.2).

Case 1. Here we have $|\mathbf{N}(S)|=3^{3} \cdot 7$ and $|\mathbf{N}(T)|=2^{2} \cdot 3^{3}$. By assumption, an element of order 7 is centralized by a group of order 9 . If $T^{\prime} \neq\langle 1\rangle$, then $T^{\prime}$ is centralized by an involution and by an element of order 7 which is against (3.1). Thus $T$ is abelian. Note that a $S_{2}$-subgroup of $\mathbf{N}(T)$ has order 4. Application of (3.1) yields that a $S_{2}$-subgroup of $\mathbf{N}(T)$ is conjugate in $G$ to $\left\langle z_{1}, d\right\rangle$ and to $\left\langle a, b^{\prime}\right\rangle$. But this contradicts the fact that $z_{1} \sim d \sim z_{1} d$ and $a$ is not conjugate to $z_{1}$ in $G$. Case 1 is ruled out.

Case 2. Here we have $|\mathbf{N}(S)|=3^{3} \cdot 7$ and $|\mathbf{N}(T)|=3^{3}$. From a transfer result of Burnside we get $T^{\prime} \neq\langle 1\rangle$. By assumption, an element of order 7 centralizes a subgroup of order 9 . Thus, we may assume that $\mathrm{C}(S)=S \times R$, where $R$ has order 9 and $R \subset T$. Evidently, $T^{\prime} \subset R \subset T$. Put $S=\langle\sigma\rangle$ and $T^{\prime}=\langle\xi\rangle$. As $\mathbf{N}(T)=T$, we see that $\xi$ is not conjugate to its inverse; clearly, $\xi$ is not centralized by an involution. Thus, $|C(\xi)|=3^{3} \cdot 7$. There are six $G$-conjugates of $c$ and six $G$-conjugates of $\gamma$ in $T$. Therefore, $T$ is generated by elements of order 3. From [3, 5.3.9] it follows that $T$ has exponent 3. Since $\left|\mathrm{Aut}_{G}(S)\right|=3$, we see that $\sigma$ is not conjugate to $\sigma^{-1}$ in $G$. Let $x, y$ be in $R^{\#}$ and $x \neq y$. Then, $x \sigma$ and $y \sigma$ have order 21 and are not conjugate in $G$, since such a conjugation would be performed in $\mathbf{C}(\sigma)$ which is abelian. Also, $x \sigma, x \sigma^{-1}$, and $y \sigma^{-1}$ lie in three pairwise different $G$-classes as $\left|\operatorname{Aut}_{G}(S)\right|=3$. It follows that in $S \times R$ there are representatives for $16 G$-classes of elements of order 21 . If $x \in R^{\#}$, then $|\mathbf{C}(x \sigma)|=\left|\mathbf{C}\left(x \sigma^{-1}\right)\right|=3^{2} \cdot 7$. Our assumptions imply that $|\mathbf{C}(c)|=|\mathbf{C}(\gamma)|=$ $2 \cdot 3^{2}$. As the centralizers of roots of involutions are known, we may write down the conjugacy classes of $G$ discussed so far, and we see that $G$ has at least 13,056 elements. Since $|G|=12,096$, we have shown that Case 2 does not occur.

Case 3. Here we have $|\mathbf{N}(S)|=2 \cdot 3 \cdot 7$ and $|\mathbf{N}(T)|=2^{2} \cdot 3^{3}$. If $T$ were abelian then we would get from (3.1) that $\left\langle z_{1}, d\right\rangle$ and $\left\langle a, b^{\prime}\right\rangle$ are conjugate in $G$ which, however, is not the case. Therefore, $T^{\prime} \neq\langle 1\rangle$ and $T^{\prime}$ is centralized by an involution. Since $T^{\prime} \sim\langle c\rangle$ or $T^{\prime} \sim\langle\gamma\rangle$, there is a four-subgroup $V$ in $\mathbf{N}(T)$. Acting with $V$ on appropriate $V$-admissible 
sections of $T / T^{\prime}$, we see that $T=\Omega_{1}(T)$. Application of $[3,5.3 .9]$ yields that $\exp (T)=3$.

Put $\mathbf{N}(T)=T\langle\alpha, \beta\rangle$, where $\langle\alpha, \beta\rangle$ is a four-subgroup of $G$. Then, all involutions of $\langle\alpha, \beta\rangle$ are conjugate in $G$ but fall into three $\mathbf{N}(T)$-classes. We know that $\left|\mathbf{C}_{T}(\alpha)\right|=\left|\mathbf{C}_{T}(\beta)\right|=\left|\mathbf{C}_{T}(\alpha \beta)\right|=3$. Without loss of generality we may set $T^{\prime}=\mathbf{C}_{T}(\alpha)$. The groups $\mathbf{C}_{T}(\beta)$ and $\mathbf{C}_{T}(\alpha \beta)$ are not conjugate in $\mathbf{N}(T)$, since $\beta \nsim \alpha \beta$ holds in $\mathbf{N}(T)$. It follows that a generator for $\mathbf{C}_{T}(\beta)$ has precisely six conjugates in $\mathbf{N}(T)$; the same is true for a generator for $C_{T}(\alpha \beta)$. So far, we have got 14 elements of order 3 in $T$ which are conjugate to an element of order 3 in $\mathbf{C}_{G}(\alpha)$. There is an element of order 3 in $T$ which is centralized by an involution which is not conjugate to $\alpha$. Such an element has precisely 12 conjugates in $\mathbf{N}(T)$. It follows that $G$ has precisely two classes of elements of order 3. In particular, either $c$ or $\gamma$ is 3-central in $G$.

Writing down the complete table of the conjugacy classes of $G$, we get $|G|=10,752$. Therefore, Case 3 is ruled out.

Case 4. Here we have $|\mathbf{N}(S)|==2 \cdot 3 \cdot 7$ and $\mathbf{N}(T)=T$. By a result of Burnside it is clear that $T^{\prime} \neq\langle 1\rangle$. Hence $T^{\prime}=\mathbf{Z}(T)$ has order 3. We have shown above that $|\mathrm{C}(S)|=7$. Clearly, $T^{\prime}$ is not centralized by an involution and $\left|C\left(T^{\prime}\right)\right|=3^{3}$.

Consider $\mathbf{N}(\langle c\rangle)$. This group has order $2^{2} \cdot 3^{2}$ and $\left\langle z_{1}, d\right\rangle$ as a $S_{2}$ subgroup with $z_{1} \sim d \sim z_{1} d$ in $G$ and $d c d=c^{-1}$. From (3.1) and the order of $H$ we get that the $S_{3}$-subgroup of $\mathrm{N}(\langle c\rangle)$ is elementary abelian. Let $\langle t, c\rangle$ be the subgroup of order 9 of $\mathbf{N}(\langle c\rangle)$. We may assume that $t$ is 3-central in $G$. Thus $\langle t\rangle$ is not normalized by a 2-subgroup of $G$ different from $\langle 1\rangle$. In particular, $t \nsim t^{-1}$ in $G$. It follows that $t$ and $t^{-1}$ each have four conjugates in $\mathbf{N}(\langle c\rangle)$. But $c$ is not 3-central and has precisely two conjugates under the action of $\mathbf{N}(\langle c\rangle)$. Since $1+2+4+4=11>9$, we have obtained a contradiction which shows that Case 4 does not occur.

Summarizing we get

(3.3) Lemma. The case $L \cong \Sigma_{4}$ does not occur.

\section{The case $L \cong \Sigma_{5}$}

From (3.3), (2.7), and (2.12) we conclude that $L \cong \Sigma_{5}$ and that $|G|=$ $2^{6} \cdot 3^{3} \cdot 5 \cdot 11$.

It is our aim to determine the structures of all Sylow normalizers and to write down the uniquelydetermined table of the conjugacy classes for $G$. 
Further, we are interested in the normalizers of certain elementary subgroups of order 9 of $G$.

(4.1) Lemma. We have $\mathrm{C}(c)=K_{c}\left\langle z_{1}\right\rangle$, where $K_{c}$ is a normal subgroup of $\mathbf{C}(c)$ of order $3^{2}$ or $3^{3}$, and $\mathbf{N}(\langle c\rangle)=K_{c}\left\langle z_{1}, d\right\rangle$. The four-group $\left\langle a, b^{\prime}\right\rangle$ is a $S_{2}$-subgroup of $\mathbf{C}(\gamma)$.

Proof. Clearly $\left\langle z_{1}\right\rangle \in \operatorname{Syl}(\mathbf{C}(c))$. Thus $\mathbf{C}(c)$ has a normal 2-complement $K_{c}$. Now, $\left\langle z_{1}, d\right\rangle$ acts on $K_{c}$ and all involutions of $\left\langle z_{1}, d\right\rangle$ are conjugate in $G$. Thus, the first assertion follows from $[9,5.1 .9]$ and the Frattini argument. As for the second assertion, note that all involutions of $\left\langle a, b^{\prime}\right\rangle$ are conjugate in $G$.

(4.2) Lemma. A Sylow 5-normalizer of $G$ is contained in $\mathbf{C}(a)$.

Proof. Denote by $w$ an element of order 5 of $\mathbf{C}(a)$. We have $\mathbf{C}(a) \cap$ $\mathbf{C}(w)=\langle a\rangle \times\langle w\rangle$, and so $\langle a\rangle$ is a $S_{2}$-subgroup of $\mathbf{C}(w)$. Thus $\mathbf{C}(w)$ possesses a normal 2-complement $K$. There is an involution $z \in L^{\prime}$ inverting $w$. Therefore, from the action of $\langle a, z\rangle$ on $K$ and from $[9,5.1 .9]$ together with the Frattini argument and (4.1) we get that $|K| \in\left\{5,3 \cdot 5,3^{2} \cdot 5\right\}$. From the structure of $\mathbf{C}(a)$ follows that in $G$ there is precisely one class of elements of order 5. Thus $\mathbf{N}(\langle w\rangle) / \mathbf{C}(w) \cong Z_{4}$. Since $|\mathbf{C}(w)|=2 \cdot|K|$, we get from Sylow's theorem that $\mathbf{C}(w)=\langle a\rangle \times\langle w\rangle$. The assertion follows. 55.

(4.3) Lemma. A Sylow 11-normalizer of $G$ is a Frobenius group of order

Proof. Denote by $e$ an element of order 11 in $G$. We know that $|\mathrm{C}(e)|$ is neither divisible by 2 nor by $3^{3}$ as $\left|K_{c}\right| \in\left\{3^{2}, 3^{3}\right\}$. Also, there are no elements of order 55 in $G$. Thus $|C(e)| \in\left\{11,3 \cdot 11,3^{2} \cdot 11\right\}$. From a transfer result we get $\left|\operatorname{Aut}_{G}(\langle e\rangle)\right| \in\{2,5,10\}$. Application of Sylow's theorem yields $|\mathbf{N}(\langle e\rangle)|=5 \cdot 11$. The assertion follows.

(4.4) Lemma. $A S_{3}$-subgroup of $G$ is nonabelian.

Proof. Assume by way of contradiction that a $S_{3}$-subgroup $T$ of $G$ is abelian. Since by (4.1) a four-group acts on $T$, we get that $T$ is elementary abelian. From the above results we get $\mathbf{C}(T)=T$ and $\mathbf{N}(T) \supset T$. Since $T$ cannot have automorphisms of order 5 or 11 , we get that $\mathbf{N}(T) / T$ is a 2group. From the order of $G L(3,3)$ it follows that $|\mathbf{N}(T) / T| \leq 2^{5}$. Sylow's theorem yields $|\mathbf{N}(T)| \in\left\{2^{4} \cdot 3^{3}, 2^{2} \cdot 3^{3}\right\}$. A lemma of Burnside implies 
that any two $G$-conjugate elements in $T$ are conjugate under the action of $\mathbf{N}(T)$. Let $x, y \in T$ such that $x \sim c, y \sim \gamma$ in $G$. Then $\mathbf{C}(x)$ has order $2 \cdot 3^{3}$ and lies in $\mathbf{N}(T)$. We consider first the case that $|\mathbf{N}(T)|=2^{4} \cdot 3^{3}$. Then $x$ has precisely 8 conjugates in $T$. We have $|\mathrm{C}(y)|=2^{2} \cdot 3^{3}$, and so, $y$ has 4,8 , or $16 G$-conjugates in $T$. Note that $1+8+16=25<27$. In $T^{\prime \prime}$ there must be an element $t$ which is not centralized by an involution. Thus $t$ has 16 conjugates in $\mathbf{N}(T)$. But $1+8+4+16=29>27$ gives a contradiction. Finally, consider the case $|N(T)|=2^{2} \cdot 3^{3}$. From (4.1) we get that a $S_{2}$-subgroup of $\mathbf{N}(T)$ is conjugate to $\left\langle z_{1}, d\right\rangle$ in $G$. A result of W. Gaschütz implies that $\mathbf{C}(\gamma)=\langle\gamma\rangle \times X$, where $|X|=2^{2} \cdot 3^{2}$; remember that $\left\langle a, b^{\prime}\right\rangle$ is a $S_{2}$-subgroup of $X$. Since $z_{1}$ is not conjugate to $a$ in $G$, we see that a $S_{3}$-subgroup of $\mathbf{C}(\gamma)$ is not normal in $\mathbf{C}(\gamma)$. If a minimal normal subgroup of $X$ is a 2-group or a group of order 3 , then an involution of $X$ is centralized by a subgroup of order 9 of $\mathbf{C}(\gamma)$. This contradiction proves the lemma.

(4.5) LEMMA. $A S_{3}$-subgroup $T$ of $G$ is nonabelian of exponent 3 and $|\mathbf{N}(T)|=2^{2} \cdot 3^{3}$.

Proof. We know that $\mathrm{C}(T)=T^{\prime}=\mathbf{Z}(T)$ from (4.1). For the order of $|\mathbf{N}(T)|$ we get the following possibilities from Sylow's theorem: $3^{3}, 2^{2} \cdot 3^{3}$, $2^{4} \cdot 3^{3}$. The case $|N(T)|=2^{4} \cdot 3^{3}$ is not possible as an element of order 3 is not centralized by a group of order 8 .

Let us assume $|\mathbf{N}(T)|=3^{3}$. Put $T^{\prime}=\langle\xi\rangle$. Then, by a lemma of Burnside, $\xi$ is not inverted in $G$, and so, $\xi$ is neither conjugate to $c$ nor to $\gamma$ in $G$. Thus, in $G$ there are at least four classes of elements of order 3.

To obtain a contradiction we write down the table for the conjugacy classes of $G$ obtained so far, and we get at least 105,600 elements in $G$. Thus, the case that $|\mathbf{N}(T)|=3^{3}$ is ruled out.

We are left with the case $|\mathbf{N}(T)|=2^{2} \cdot 3^{3}$. Since $T^{\prime} \neq\langle 1\rangle$, we get $T^{\prime} \sim\langle c\rangle$ or $T^{\prime} \sim\langle\gamma\rangle$. From the structure of $\mathbf{C}(a)$ it follows that a $S_{2}$-subgroup of $\mathbf{N}(\langle\gamma\rangle)$ is elementary of order 8 . Thus, a $S_{2}$-subgroup of $\mathbf{N}(T)$ is elementary abelian of order 4. From the orders of the centralizers of involutions it follows that $T$ is generated by elements of order 3 . Since the nilpotency class of $T$ is 2 , application of $[3,5.3 .9]$ yields that $T$ has exponent 3 . The lemma is proved.

(4.6) Lemma. The element $c$ is 3-central.

Proof. Assume that there is a $S_{3}$-subgroup $T$ of $G$ such that $T^{\prime}=$ $\langle\gamma\rangle$. Then $\mathbf{C}(\gamma)=T\left\langle a, b^{\prime}\right\rangle$. Consider the factor group $X=T\left\langle a, b^{\prime}\right\rangle /\langle\gamma\rangle$. 
From the orders of the centralizers of involutions and by the stabilizing-chain argument [3,5.3.2], we get that no involution of $X$ centralizes an element of order 3 of $X$. As this is not possible, we get that $c$ is 3-central.

We are able to write down the uniquely determined table of the conjugacy classes of the simple group $G$ of order 95,040 .

TABLE IV

\begin{tabular}{cccc}
\hline$x$ & $o(x)$ & $\left|\mathrm{C}_{G}(x)\right|$ & $\left|c c l_{G}(x)\right|$ \\
\hline 1 & 1 & 95,040 & 1 \\
$z_{1}$ & 2 & $2^{6} \cdot 3$ & 495 \\
$a$ & 2 & $2^{4} \cdot 3 \cdot 5$ & 396 \\
$z_{3} a$ & 4 & $2^{5}$ & 2970 \\
$z_{2} z_{3} a$ & 4 & $2^{5}$ & 2970 \\
$b d$ & 8 & $2^{3}$ & 11880 \\
$z_{3} b d$ & 8 & $2^{3}$ & 11880 \\
$c$ & 3 & $2 \cdot 3^{3}$ & 1760 \\
$z_{1} c$ & 6 & $2 \cdot 3$ & 15840 \\
$\gamma$ & 3 & $2^{2} \cdot 3^{2}$ & 2640 \\
$a \gamma$ & 6 & $2^{2} \cdot 3$ & 7920 \\
$w$ & 5 & $2 \cdot 5$ & 9504 \\
$a w$ & 10 & $2 \cdot 5$ & 9504 \\
$e_{1}$ & 11 & 11 & 8640 \\
$e_{2}$ & 11 & 11 & 8640 \\
\hline
\end{tabular}

We see that Table IV is identical with the table for the Mathieu group $M_{12}$.

(4.7) Lemma. Let $T \in \operatorname{Syl}_{3}(G)$ such that $T^{\prime}=\langle c\rangle$. Then, $\mathbf{N}(T)=$ $T\left\langle z_{1}, d\right\rangle$ and $\mathbf{N}(T)$ contains elementary abelian subgroups $M_{1}, M_{2}$ such that $\mathbf{N}\left(M_{i}\right)$ is a splitting extension of $M_{i}$ by $G L(2,3)$ for $i=1,2$. Further, $M_{1}$ is not conjugate to $M_{2}$ in $G$. There are two elementary abelian subgroups of order 9 in $T$ which are conjugate in $\mathrm{N}(T)$ and have only two 3-central elements each.

Proof. Clearly $\mathbf{N}(T)=T\left\langle z_{1}, d\right\rangle$. Every involution of $\left\langle z_{1}, d\right\rangle$ is conjugate to $z_{1}$ in $G$ and centralizes a subgroup of order 3 of $T$; clearly $T=\mathbf{C}_{T}\left(z_{1}\right) \cdot \mathbf{C}_{T}(d) \cdot \mathbf{C}_{T}\left(z_{1} d\right)$. Put $\mathbf{C}_{T}(d)=\langle r\rangle, \mathbf{C}_{T}\left(z_{1} d\right)=\langle s\rangle$. Then 
$c \sim r \sim s$ holds in $G$. Put $M_{1}=\langle c, r\rangle$ and $M_{2}=\langle c, s\rangle$. Evidently, $M_{i}$ is normal in $\mathbf{N}(T)$, and so, $M_{i}^{*}$ consists only of 3-elements which are 3-central. In $T$ there are 12 conjugates of $\gamma$, and this implies that $M_{1} \cup M_{2}$ contains all the 14 3-central elements of $T$. Clearly, $\langle c, r s\rangle \sim\left\langle c, r s^{-1}\right\rangle$ via $d$ and $M_{1}$ is not conjugate $M_{2}$ by a result of Burnside.

Since $M_{i}$ possesses precisely four subgroups of order 3 , we get that $M_{i}$ is normalized by precisely four $S_{3}$-subgroups of $G$, one of which is $T$. Moreover, $\left\langle z_{1}, d\right\rangle$ normalizes $M_{i}$. Since $\mathbf{C}\left(M_{i}\right)=M_{i}$, we see that $\mathbf{N}\left(M_{i}\right)$ is a $(2,3)$-group. As $\left|\mathbf{N}\left(M_{i}\right): \mathbf{N}\left(M_{i}\right) \cap \mathbf{N}(T)\right|=4$, we get $\left|\mathbf{N}\left(M_{i}\right)\right|=2^{4} \cdot 3^{3}$. It follows that $\mathbf{N}\left(M_{i}\right)$ is a splitting extension of $M_{i}$ by $G L(2,3)$ as $T$ has exponent $3 ; i=1,2$.

\section{The identification of $G$ with $M_{12}$}

In what follows we shall change our notation completely, because we are going to find generators and relations for $G$ as given in [11, p. 421]. So, we shall use from now on only the structural information obtained for $G$ so far.

There is an elementary abelian subgroup $M$ of order 9 of $G$, the normalizer of which is a splitting extension of $M$ of $G L(2,3)$. Since $\mathbf{C}(M)=M$, we get that $\mathbf{N}(M)$ is uniquely determined.

Studying Todd's presentation for $M_{12}$, we see that we may put $\mathbf{N}(M)=$ $\left\langle a^{2} c, a c a\right\rangle\langle a, b, e, f\rangle$ so that the relations between the generators of $\mathbf{N}(M)$ are those of Todd. Then, we have $M=\left\langle a^{2} c, a c a\right\rangle$ and $\langle a, b, e, f\rangle \cong$ $G L(2,3)$.

Clearly, $\langle a, b, e, f\rangle$ lies in $\mathbf{C}\left(a^{2}\right)=H$ and $a^{2}$ is a 2-central involution. Since $e f$ is an element of order 3 in $H$, it follows $f \in H \backslash O_{2}(H)$. We know that $\langle a, b\rangle$ is a normal quaternion subgroup of $H$. We may thus add a generator $d$ for $H$ so that Todd's relations hold between the generators of $H$. We get $H=\langle a, b, d, e, f\rangle$ and $\langle d, e, f\rangle \cong \Sigma_{4}$.

Thus, to prove $G \cong M_{12}$, it suffices to show that $(c d)^{3}=1$.

Consider the diagram

$$
\circ a^{2} \circ \frac{m}{c} \circ-\circ-\circ
$$

All relations represented by the diagram are known except $(c d)^{m}=1$. It is easy to see that all involutions occurring in the diagram are 2-central in $G$.

We have $\left\langle a^{2}, c, d\right\rangle \subseteq \mathbf{C}(f)=H_{f}$. Since $o\left(a^{2} c\right)=3$, we get that both $a^{2}$ and $c$ are contained in $H_{f} \backslash O_{2,3}\left(H_{f}\right)$. The table of conjugacy classes of $H$ shows that all involutions of $H \backslash O_{2}(H)$ are conjugate in $H$. It follows that 
$\mathrm{C}\left(a^{2}\right) \cap H_{f}=\left\langle f, x_{2}, a^{2}\right\rangle$ is elementary abelian of order 8 , where $\left\langle f, x_{2}, x_{3}\right\rangle$ is the normal elementary abelian subgroup of order 8 of $H_{f}$.

Now $d$ lies in $\left\langle f, x_{2}, a^{2}\right\rangle$. Assume first that $d \in\left\langle f, x_{2}\right\rangle$. Then $c d \in$ $\left\langle f, x_{2}, x_{3}\right\rangle c$, and so the order of $c d$ is 2 or 4 .

Assume next that $d \in\left\langle f, x_{2}, a^{2}\right\rangle \backslash\left\langle f, x_{2}\right\rangle$. Then, $d=a^{2} x$ with $x \in$ $\left\langle f, x_{2}\right\rangle$. It follows $c d \in c a^{2}\left\langle f, x_{2}, x_{3}\right\rangle$, and as $o\left(c a^{2}\right)=o\left(a^{2} c\right)=3$, we get that $o(c d) \in\{3,6\}$. If $o(c d)=6$, then the structure of $H$ shows that $(c d)^{3}=f$.

We have thus obtained the following possibilities for $m: m \in\{2,4,3,6\}$.

First we shall eliminate the possibility $m=2$. This is easy as the assumption $o(c d)=2$ implies that in $G$ the subgroup $\langle d, e, f\rangle$ which is isomorphic to $\Sigma_{4}$ is centralized by the element $a^{2} c$ of order 3 . But this contradicts the results of Table IV.

Next, we shall rule out the case $m=6$. Assume that $o(c d)=6$. Put $A=\langle c, d, e, f\rangle$. The generators of $A$ respect the diagram

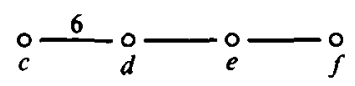

plus the additional relation $(c d)^{3} f=1$. Compute

$$
(c d)^{e d e c}=c^{e d e c} d^{e d e c}=c^{d e c} e=c e d c d e c e=e c d c d c=e f d .
$$

It follows that the element $e f d$ of order 6 lies in the subgroup $\langle d, e, f\rangle$ of $G$ which is isomorphic to $\Sigma_{4}$. This is a contradiction which shows that the case $m=6$ is not possible.

Finally, we treat the case $o(c d)=4$. Put $A=\left\langle a^{2}, c, d, e\right\rangle$ and $Y=$ $\left\langle a^{2}, c, d\right\rangle$. The generators of $A$ respect the diagram

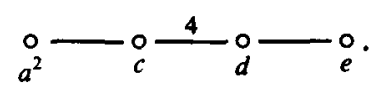

Therefore, $A$ is an epimorphic image of the Coxeter group $F_{4}$ (see, for example, [2, Table 10]) and so, $|A|$ divides $2^{6} \cdot 3^{2}$. We know that $Y$ has order divisible by $2^{3} \cdot 3$ and that $Y$ is an epimorphic image of $Z_{2} \times \Sigma_{4}$; see [2, Table 10]. Thus, $Y$ is isomorphic to $\Sigma_{4}$ or to $Z_{2} \times \Sigma_{4}$. The case $|A|=24$ is not possible as then $Y=A$. But in $\Sigma_{4}$ there is no element of order 6. If $|A|=2^{4} \cdot 3$, then $A \cong Z_{2} \times \Sigma_{4}$ which would imply $e \in Z(A)$ as $e$ centralizes the element $a^{2} c$ of order 3 of $A$. Thus, if $3^{2}$ does not divide $|A|$, then $|A|=2^{6} \cdot 3$ as $\left|\mathbf{N}_{A}\left(\left\langle a^{2} c\right\rangle\right)\right|=2^{2} \cdot 3$; note that $a^{2} c$ is 3 -central in $G$. If $|A|=2^{6} \cdot 3$, then $\left|O_{2}(A)\right|=2^{5}$, and the element $a^{2} c$ centralizes the 
involution $e$ which must lie in $O_{2}(A)$. This contradicts the fact that $d e$ has order 3.

Assume now that $3^{2}$ divides $|A|$. Then $2^{3} \cdot 3^{2}$ divides $|A|$ and since $\Sigma_{4}$ is present, we see that $A$ is not 3-closed.

First suppose that $|A|=2^{3} \cdot 3^{2}$. A Sylow 2-subgroup of $A$ is dihedral. If $O_{3}(A)=\langle 1\rangle$, then $O_{2}(A)$ is a four-group which is centralized by a group of order 3, contradicting $O_{3}(A)=\langle 1\rangle$. Thus, $O_{3}(A)$ has order 3 . It follows that $A \cong\left(Z_{3} \times A_{4}\right) Z_{2}$ with $A_{4} Z_{2} \cong \Sigma_{4}$. But then, a non-2-central involution of $G$ would have a root of order 4 which is not the case.

We have proved that $2^{4} \cdot 3^{2}$ divides $|A|$. Let $X$ be a minimal normal subgroup of $A$. Then $A$ is not a 3-group as $A$ is not 3-closed and no element of order 3 of $G$ is centralized by a subgroup of order 8 . Hence, $|X| \in\left\{2,2^{2}, 2^{3}\right\}$. From the structures of centralizers of involutions of $G$ we get that $X$ is a four-group. As $X$ is centralized by an element of order 3 we get that all elements of $X^{\#}$ are non-2-central. Since a non-2-central involution of $G$ has no roots of order 4, we see that all involultions of $A$ centralize $X$. This implies that $\left\langle a^{2}, c\right\rangle$ centralizes $X$. But this is a contradiction, since $a^{2} c$ is 3-central. The case $m=4$ has been ruled out.

It remains $o(c d)=3$. Thus $G$ possesses elements $a, b, c, d, e, f$ which satisfy the Todd relations for a presentation of the simple Mathieu group $M_{12}$. Since $|G|=\left|M_{12}\right|$, we get $G \cong M_{12}$, and we have reached our final goal.

\section{References}

[1] R. Brauer and P. Fong, 'A characterization of the Mathieu group $M_{12}$,' Trans. Amer. Math. Soc. 122 (1966), 18-47.

[2] H. S. M. Coxeter and W. O. J. Moser, Generators and relations for discrete groups, (Springer-Verlag, Berlin, Göttingen, Heidelberg, 1957).

[3] D. Gorenstein, Finite groups, (Harper \& Row, New York, Evanston, and London, 1968).

[4] D. Held, 'A characterization of some multiply transitive permutation groups, II.' Arch. Math. 19 (1968), 378-382.

[5] D. Held, 'The simple groups related to $M_{24}$,'J. Algebra 13 (1969), 253-296.

[6] D. Held and J. Hrabě de Angelis, 'A block-theory-free characterization of $M_{24}$,' Rend. Sem. Mat. Univ. Padova 82 (1989), to appear.

[7] D. Held and J. Hrabè de Angelis, 'A character-theory-free characterization of the simple groups $M_{11}$ and $L_{3}(3)$,' to appear.

[8] R. G. Stanton, 'The Mathieu groups,' Canad. J. Math. 3 (1951), 164-174.

[9] M. Suzuki, Group theory II, (Springer-Verlag, New York, Berlin, Heidelberg, Tokyo, 1986).

[10] J. G. Thompson, 'Nonsolvable finite groups all of whose local subgroups are solvable,' Bull. Amer. Math. Soc. 74 (1968), 383-437. 
[11] J. A. Todd, 'Abstract definitions for the Mathieu groups,' Quart. J. Math. Oxford (2) 21 (1970), 421-424.

[12] W. J. Wong, 'A characterization of the Mathieu group $M_{12}$, Math. Z. 84 (1964), 378388.

Fachbereich Mathematik

Universität Mainz

D-6500 Mainz

Federal Republic of Germany 